\title{
Tödliche Risiken entschärfen
}

\section{Einfluss einer ballaststoffreichen Ernährung auf die Häufigkeit von Herz-Kreislauf- Erkrankungen und Krebs: Möglichkeiten und Perspektiven}

\author{
Reducing Deadly Risks \\ The Influence of a Fibre-Rich Diet on the Risk of Cardiovascular Diseases and Cancer: Possibilities and Perspectives
}

Autor

Institut

\section{H. Boeing}

Deutsches Institut für Ernährungsforschung, Potsdam-Rehbrücke
Schlüsselwörter

- Ernährungsepidemiologie

- Sterblichkeitsrisiko

- Todesursachen

- Dickdarmkrebs

- kardiovaskuläre Ereignisse

- Blutdruck

- Insulinstoffwechsel

- Getreideballaststoffe

\section{Keywords}

nutritional epidemiology

- mortality risk

- causes of death

- cancer of the large bowel

- cardiovascular events

- blood pressure

- insulin metabolism

- cereal fibres
Bibliografie

Dol http://dx.doi.org/

10.1055/s-0033-1360027

Aktuel Ernahrungsmed 2014;

39, Supplement 1: S25-S27

(c) Georg Thieme Verlag KG

Stuttgart - New York

ISSN 1862-0736

\section{Korrespondenzadresse}

Prof. Dr. Heiner Boeing

Abteilung Epidemiologie,

Deutsches Institut für

Ernährungsforschung (DIfE)

Arthur-Scheunert-Allee 114-

116

14558 Nuthetal

Tel.: 033200/88-2710

boeing@dife.de

\section{Zusammenfassung \\ $\nabla$}

Da Ballaststoffe viele Krankheiten beeinflussen, ist eine globale Betrachtung dieser Assoziationen sinnvoll. Eine globale Betrachtung wird u. a. durch die Untersuchung der Sterblichkeit erreicht, da sie das allgemeine Krankheitsgeschehen zusammenfasst. Krebserkrankungen und Herz-KreislaufKrankheiten sind in Deutschland die häufigsten Todesursachen. Dabei beeinflusst die Ballaststoffaufnahme die Sterblichkeit aufgrund chronischer Erkrankungen in mehrfacher Hinsicht: Dazu zählen ihr direkter Einfluss auf die Entstehungsmechanismen von Krebs und Herz-Kreislauf-Krankheiten und ihr indirekter Einfluss auf deren Risikofaktoren wie Hypertonie, Adipositas und Typ2-Diabetes.

Großen Studien zufolge ist das Sterblichkeitsrisiko bei steigender Ballaststoffzufuhr geringer. Besonders ausgeprägt ist dieser Zusammenhang bei Getreideballaststoffen. Die Ballaststoffaufnahme ist auch invers mit dem Risiko insbesondere für Kolorektalkarzinome verbunden. Ballaststoffe wirken vermutlich sowohl über lokale Effekte im Darmlumen als auch über metabolische Effekte, unter anderem auf den Glucosestoffwechsel. Auch bei den Herz-Kreislauf-Erkrankungen wie koronare Herzkrankheiten und Schlaganfall wurden inverse Zusammenhänge in Verbindung mit dem Verzehr von Ballaststoffen beobachtet.

Diese wirken vermutlich über den Blutdruck und den Insulinstoffwechsel auf das Auftreten dieser Krankheiten ein.

Ziel der Ernährungsepidemiologie ist es, gesichertes Wissen über die Ernährungs-Krankheits-Beziehungen durch den Aufbau und die Analyse von epidemiologischen Studien zur Verfügung zu stellen. Dieses Wissen trägt dazu bei, durch „Public Health“-Maßnahmen das Auftreten von Krankheiten zu reduzieren und die Lebenszeit ohne ge-

\section{Abstract \\ $\nabla$}

Since dietary fibres influence many diseases, a global view on these associations seem useful. A global view is achieved i.a. through studying mortality which summaries the general disease occurrence. Cancer and cardio-vascular diseases are the most important causes of death in Germany. Thereby, dietary fibres influence the mortality due to chronic diseases in manifold ways: With it, one can count the direct influence on cancer and cardio-vascular diseases and the indirect influence on hypertension, obesity, and type 2 diabetes.

According to large studies, mortality risk is reduced with increasing intake of dietary fibre. This association is particularly seen in case of cereal fibres. Intake of dietary fibre is also inversely associated with risk for especially colorectal cancer. Dietary fibres are probably effective through local effects in the intestinal lumen as well as through metabolic effects, among others on the glucose metabolism. Inverse associations in connection with dietary fibre intake have also been observed for cardiovascular diseases such as coronary heart disease and stroke. Dietary fibres influence the occurrence of such disorders presumably by affecting blood pressure and insulin metabolism. 


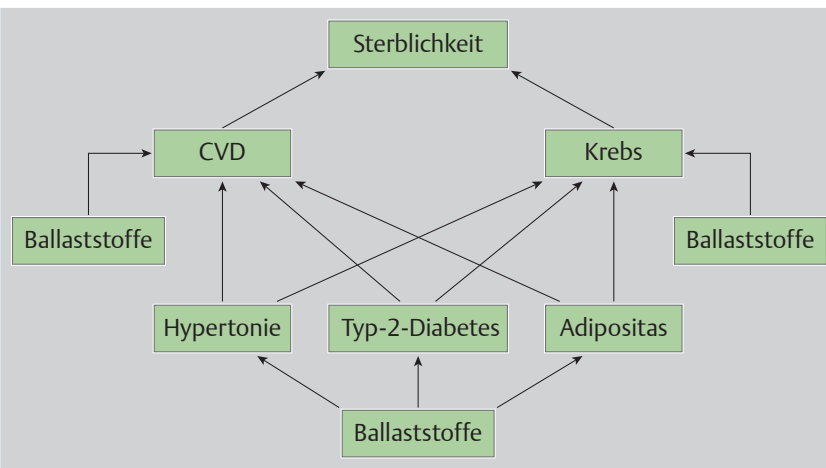

Abb. 1 Der Ballaststoffverzehr hat Auswirkungen auf das Risiko der wesentlichen an den Todesursachen beteiligten Krankheiten und deren Risikofaktoren, die dadurch wiederum das Sterblichkeitsrisiko beeinflussen.

mit einem Ernährungsverhalten beobachtetes relatives Risiko unter 1 für eine Krankheit bedeutet, dass in der Studienbevölkerung weniger Erkrankungen bei dem untersuchten Ernährungsverhalten aufgetreten sind. In Kohortenstudien besteht aufgrund des Studiendesigns die Möglichkeit, gleichzeitig viele Erkrankungen zu betrachten.

Da Ballaststoffe viele Krankheiten beeinflussen, ist eine globale Betrachtung dieser Assoziationen sinnvoll. Eine globale Betrachtung wird unter anderem durch die Untersuchung der Sterblichkeit erreicht, da sie das allgemeine Krankheitsgeschehen zusammenfasst. Die derzeit zu beobachtende Verlängerung der Lebenszeit ist ein bedeutsames Gesundheitsziel. Sie sollte aber nicht mit einer Beeinträchtigung der Gesundheit einhergehen. Ein Maß dafür sind die „Quality adjusted life years“, kurz qaly genannt.

Krebserkrankungen sind in Deutschland die häufigsten Todesursachen im mittleren bis hohen Alter, Herz-Kreislauf-Krankheiten im hohen bis sehr hohen Alter. An kardiovaskulären Ereignissen sterben 50-60\% der Betroffenen innerhalb der ersten 28 Tage, bei Krebserkrankungen liegt die Mortalität während der folgenden fünf Jahre bei circa $50 \%$.

\section{Ernährung und Sterblichkeitsrisiko \\ $\nabla$}

Anhand der Ballaststoffzufuhr kann der Zusammenhang zwischen Ernährungsverhalten und Sterblichkeit illustriert werden. Normalerweise ist es nicht unmittelbar nachvollziehbar, warum die Sterblichkeit einen Ernährungsbezug haben sollte, da die Nahrungszufuhr in den entwickelten Ländern nicht begrenzt ist und beide Ereignisse getrennt stattfinden. Ballaststoffe haben zunächst Auswirkungen auf die Erkrankungshäufigkeit von Hypertonie, Typ-2-Diabetes und Adipositas, die wiederum als Risikofaktoren für kardiovaskuläre Erkrankungen und Krebs gelten. Ballaststoffe nehmen aber auch über bestimmte Mechanismen direkt Einfluss auf kardiovaskuläre Erkrankungen und Krebs. Das Risiko für kardiovaskuläre Erkrankungen und Krebs wirkt sich auch auf das Risiko für die Sterblichkeit insgesamt aus (৫ Abb.1).

Damit ist die Sterblichkeit ein guter Parameter, um das gesamte präventive Potenzial eines Ernährungsfaktors in einer relativ einfachen Weise zu erfassen. Solche Datenanalysen ergänzen die Untersuchungen zu den einzelnen Krankheitsendpunkten, die in ihrer Zielsetzung eher ätiologisch und mechanistisch und weniger gesundheitspolitisch ausgerichtet sind.

\section{Ballaststoffzufuhr und Gesamtsterblichkeit \\ $\nabla$}

Die EPIC-Studie zeigte, dass das Sterblichkeitsrisiko mit steigender Ballaststoffzufuhr sinkt [1]. Dabei war zu beobachten, dass eine höhere Ballaststoffaufnahme als die von der DGE empfohlenen $30 \mathrm{~g}$ pro Tag keinen starken Einfluss mehr auf das Sterblichkeitsrisiko hatte. Diese Zusammenhänge zeigten sich nicht nur in der EPIC-Studie mit 519000 Teilnehmern, sondern auch in der NIH-AARP-Studie, einer Kohortenstudie der Nationalen Gesundheitsinstitute der USA mit 540000 Teilnehmern [2]. Besonders die Ballaststoffe aus Getreide führten zu einer Senkung der Gesamtsterblichkeit, aber auch der einzelnen Todesursachen, unter anderem von Herz-Kreislauf-Erkrankungen und Krebs. Das gilt sowohl für Männer als auch für Frauen. Die Assoziation ist für Getreide besonders eindrücklich, gilt aber auch für andere Ballaststoffquellen. In der Nurses Health Study wurden ebenfalls verschiedene Einflussfaktoren auf die Sterblichkeit untersucht. Mit einem reduzierten Sterberisiko assoziierte Ernährungsfaktoren waren Nüsse, mehrfach ungesättigte Fettsäuren und Ballaststoffe [3].

Ballaststoffe sind demnach ein Ernährungsfaktor, der im Sinne von „Public Health“ mit der Sterblichkeit zusammenhängt. Diese Risikobeziehung berücksichtigt unter anderem auch die Auswirkungen der Ballaststoffzufuhr auf Adipositas, Hypertonie und Typ-2-Diabetes.

\section{Ballaststoffe und Dickdarmkrebsrisiko \\ $\nabla$}

Daten der EPIC-Studie zufolge sinkt das Risiko für Dickdarmkrebs mit steigender Ballaststoffaufnahme - dokumentiert in Analysen mit unterschiedlichen Nachbeobachtungszeiten [4-6]. Die neueste Analyse [6] stammt von 2012 und umfasste 11 Jahre Nachbeobachtungszeit. Sie konnte mehr als 4500 inzidente Dickdarmkrebserkrankungen in die Analyse einschließen.

Ballaststoffe beeinflussen das Risiko für kolorektale Karzinome vermutlich zum einen durch ihre lokale Wirkung im Darmlumen. Bei ihrem Abbau entstehen leicht flüchtige Säuren wie Essig- und Buttersäure, die sich wiederum auf die Bildung von sekundären Gallensäuren und die Apoptose auswirken. Zum anderen spielen vermutlich metabolische Effekte eine Rolle, da Ballaststoffe den postprandialen Anstieg der Insulin- und Glucosekonzentration im Blut reduzieren, der sich wiederum auf die Konzentrationen der Sexual- und Wachstumshormone sowie auf die Verfügbarkeit von Transportproteinen auswirkt. Sowohl die Konzentration der Glucose als auch der Sexual- und Wachstumshormone sind mit dem Krebsrisiko assoziiert.

\section{Ballaststoffe und kardiovaskuläre Erkrankungen $\nabla$}

Auch bei Herz-Kreislauf-Erkrankungen beobachteten die dazu durchgeführten Studien inverse Zusammenhänge mit dem Verzehr von Ballaststoffen, sowohl für koronare Herzkrankheiten als auch für Schlaganfall [7-9]. Die Studien zeigen, dass nicht nur der Ballaststoffverzehr insgesamt, sondern speziell der von Vollkornprodukten mit einem reduzierten Risiko für kardiovaskuläre Ereignisse verbunden ist.

Möglicherweise sind diese Ergebnisse mit dem Einfluss von Ballaststoffen auf den Insulinstoffwechsel und die Hypertonie erklärbar. Insbesondere bei Personen mit Hypertonie sinken die 
Werte bei erhöhter Ballaststoffaufnahme [8]. Der Effekt ist dagegen geringer bei Personen mit normalem Blutdruck.

Ballaststoffe haben vermutlich keinen direkten Einfluss auf die Arteriosklerose, sondern beeinflussen eher die Risikofaktoren, vor allem das Cholesterol, den Blutdruck und die Gewichtszunahme. Eine hohe Zufuhr kann zur Senkung des Blutdrucks sowie des Gesamt- und LDL-Cholesterols im Serum führen. Außerdem verbessern sie die Insulinsensitivität und Glucosetoleranz sowohl bei Typ-2-Diabetikern als auch bei Stoffwechselgesunden.

\section{Fazit}

Insgesamt ist der Verzehr von Ballaststoffen mit dem Krankheitsgeschehen in messbarer und bedeutsamer Weise assoziiert. Eine Reduktion des Krankheitsgeschehens durch einen erhöhten Verzehr von ballaststoffreichem Getreide ist wahrscheinlich.

\section{Interessenkonflikt}

$\nabla$

Der Autor hat keinen Interessenkonflikt.

\section{Literatur}

1 Chuang $S$ - $C$ et al. Fiber intake and total and cause-specific mortality in the European Prospective Investigation into Cancer and Nutrition cohort. Am J Clin Nutr 2012; 96: 164-174

2 Park $Y$ et al. Dietary fiber intake and mortality in the NIH-AARP diet and health study. Arch Intern Med 2011; 171: 1061 - 1068

3 Baer HJ et al. Risk Factors for Mortality in the Nurses' Health Study: A Competing Risks Analysis. Am J Epidemiol 2011; 173: 319-329

4 Bingham $S$ et al. Dietary fibre in food and protection against colorectal cancer in the European Prospective Investigation into Cancer and Nutrition (EPIC): an observational study. Lancet 2003; 361: 1496-1501

5 Bingham $S$ et al. Is the association with fiber from foods in colorectal cancer confounded by folate intake? Cancer Epidemiol Biomarkers Prev 2005; 14: $1552-1556$

6 Murphy $\mathrm{N}$ et al. Dietary Fibre Intake and Risks of Cancers of the Colon and Rectum in the European Prospective Investigation into Cancer and Nutrition (EPIC). PLoS ONE 2012; 7: e39361

$7 \mathrm{Ye} E \mathrm{EQ}$ et al. Greater Whole-Grain Intake Is Associated with Lower Risk of Type 2 Diabetes, Cardiovascular Disease, and Weight Gain. J Nutr 2012; 142: 1304-1313

8 Sanchez-Muniz FJ. Dietary fibre and cardiovascular health. Nutr Hosp 2011; 27: $31-45$

9 Threapleton $D E$ et al. Dietary Fiber Intake and Risk of First Stroke: A Systematic Review and Meta-Analysis. Stroke 2013; 44: 1360-1368 